\title{
Extraction of parton distributions from lattice QCD
}

\author{
W. Detmold \\ Department of Physics, University of Washington, Box 351560, Seattle, WA 98195, U.S.A. \\ W. Melnitchouk \\ Jefferson Lab, 12000 Jefferson Avenue, Newport News, VA 23606, U.S.A.
}

A. W. Thomas

Special Research Centre for the Subatomic Structure of Matter, and Department of Physics and Mathematical Physics, University of Adelaide, Adelaide, SA 5005, Australia.

\begin{abstract}
We review the calculation of moments of both the polarized and unpolarized parton distribution functions of the nucleon in lattice QCD, and in particular their extrapolation to the physical region. We also discuss the reconstruction of the $x$ dependence of the valence quark distributions in the nucleon from a finite number of lattice moments.
\end{abstract}

\section{Introduction}

One of the defining features of any hadron are its quark and gluon (or generically, parton) momentum distributions. Considerable information has been accumulated on parton distribution functions (PDFs) of the nucleon and nuclei from deep inelastic scattering and other high energy experiments. Information from the Drell-Yan reaction in $\pi N$ scattering has also been used to determine PDFs of the pion.

Theoretically, the operator product expansion (OPE) in QCD allows one to isolate the soft, non-perturbative PDFs from the hard, perturbatively calculable scattering processes. This factorization property gives rise to a universal set of PDFs which can be used to describe various reactions. Because PDFs parameterize the interactions of quarks and gluons in the hadron over all distance scales, they are sensitive to the dynamics of QCD in the strong coupling regime - i.e., at scales $Q^{2} \sim \Lambda_{\mathrm{QCD}}^{2}$. The only rigorous theoretical approach currently capable of calculating observables in the non-perturbative regime from first principles is lattice QCD.

The first exploratory studies of PDFs on the lattice were made almost two decades ago 1 Advances in computing power have recently enabled dedicated simulations to be undertaken which for the first time can be directly compared with phenomenology 2345] Since the numerical simulations are performed in Euclidean space-time, it is not possible to compute the PDFs directly as a function of the light-cone momentum fraction, $x$ (PDFs are formally defined as light-cone correlation functions involving currents with space-time separation $\left.z^{2}-(c t)^{2} \approx 0\right)$. On 
the other hand, using the OPE one can formally express the moments of PDFs in terms of matrix elements of local operators between hadron states. These matrix elements can be calculated numerically on the lattice.

Despite the impressive recent progress in the numerical simulations, several approximations are still necessary in order to relate the lattice moments to experiment. Firstly, since space-time is discretized on the lattice, with some finite lattice spacing $a$, the results must be extrapolated to the continuum limit, $a \rightarrow 0$. The finite number of lattice sites also means that an extrapolation to the infinite volume limit is necessary to avoid omitting important physics arising from the long-range part of the nucleon wave function. In addition, since the computational cost of simulations scales with the quark mass roughly as $m_{q}^{-4}$, current lattice simulations are performed with unphysically large masses for $u$ and $d$ quarks, typically $m_{q}^{\text {latt }}>30 \mathrm{MeV}$, so that an extrapolation to physical masses, $m_{q}^{\text {phys }} \approx 5 \mathrm{MeV}$, is essential. 6

The chiral $\left(m_{q}\right)$ extrapolation is found to play a particularly vital role in understanding the connection between the lattice results and phenomenology. Whereas the lattice simulations yield results 23 for the moments of unpolarized quark distributions in the nucleon which are typically 50\% larger than experiment when extrapolated linearly to $m_{q}^{\text {phys }}$, inclusion of the non-linear dependence on $m_{q}$ arising from the long-range structure of the nucleon removes most of the discrepancy! 7 Furthermore, significant finite volume effects have been found ${ }^{5}$ in the calculation of the axial vector charge $g_{A}$, which may eventually explain the residual discrepancy between the extrapolated lattice value and experiment.

In this mini-review we focus on the challenges involved with extrapolating the lattice data from the currently accessible regions of parameter space to the physical region. Using constraints available from chiral effective theory, in Sec. 2 we review extrapolation formulas which seek to describe the lattice data over a large range of quark masses, and in the chiral limit. The moments of both the spin-averaged and spin-dependent quark distributions in the nucleon are considered (a corresponding analysis of the moments of the quark distributions in the pion was also undertaken recently ${ }^{8}$ ). The more ambitious task of reconstructing the $x$ dependence of PDFs from a finite number of moments is discussed in Sec. 3. Because of the small number of lattice moments available, only valence distributions can be analyzed at present; sea quark distributions await future lattice data on higher moments of PDFs which are necessary for an independent determination of the valence and sea components. Finally, in Sec. 4 we draw some conclusions, and outline prospects for the reconstruction of PDFs from future lattice simulations. 


\section{Extracting physical results from lattice simulations}

The moments of parton distributions in the nucleon are formally defined as

$$
\begin{aligned}
\left\langle x^{n}\right\rangle_{q} & =\int_{0}^{1} d x x^{n}\left[q(x)-(-1)^{n} \bar{q}(x)\right], \\
\left\langle x^{n}\right\rangle_{\Delta q} & =\int_{0}^{1} d x x^{n}\left[\Delta q(x)+(-1)^{n} \Delta \bar{q}(x)\right], \\
\left\langle x^{n}\right\rangle_{\delta q} & =\int_{0}^{1} d x x^{n}\left[\delta q(x)-(-1)^{n} \delta \bar{q}(x)\right],
\end{aligned}
$$

for the spin-averaged, helicity and transversity distributions, respectively. From their definition, the moments alternate between the total $(q+\bar{q})$ and valence $(q-\bar{q})$ distributions, depending on whether $n$ is even or odd. Using the OPE, these moments can be related to ground state hadron matrix elements of specific twist-two operators, $\sqrt{9}$ which are calculated on the lattice.

In this review we focus not on the details of the lattice simulations of the moments (a comprehensive survey of results was recently given by the authors $\frac{10}{\text { ) }}$, but on their physical interpretation. In particular, we examine the consequences of chiral symmetry for the extrapolation of PDF moments to physical values of the quark mass, $m_{q}$.

\subsection{Chiral symmetry}

The importance of chiral symmetry and the role of the pion cloud in hadronic physics is well known!1112 At small quark masses, hadronic observables can be systematically expanded in a series in $m_{q}$ within the framework of chiral perturbation theory $(\chi \mathrm{PT})$. While the expansion coefficients are generally free parameters, one of the unique consequences of spontaneous chiral symmetry breaking in QCD is the appearance of terms involving odd powers or logarithms of $m_{\pi}$. From the GellMann-Oakes-Renner relation, which relates the quark and pion masses at small $m_{\pi}, m_{\pi}^{2} \sim m_{q}$, one finds that such terms are non-analytic in the quark mass. Furthermore, their coefficients, which are determined from the infrared behavior of the pion loops, are generally model independent.

The non-analytic term involving the lowest power of $m_{\pi}$ is known as the "leading non-analytic" (LNA) term in the expansion. For the moments of the nucleon PDFs this was shown by Thomas et al $\frac{13}{13}$ to have the generic behavior $m_{\pi}^{2} \log m_{\pi}$ arising from $\pi N$ intermediate states. This was later confirmed in $\chi \mathrm{PT}$, where the coefficients of these terms were also calculated, both for the nucleon, 1415 and for the pion 14 Using these constraints, a low order chiral expansion for the moments of the PDFs was developed recently by Detmold et al.,78101617 which incorporated the LNA behavior of the moments as a function of $m_{q}$ and also provided a connection to the heavy quark limit. 


\subsection{Chiral extrapolation of PDF moments}

Direct comparison of the currently available lattice moments of nucleon PDFs with phenomenological ones is non-trivial. The lattice moments in general receive contributions from diagrams in which the local operator insertions are on quark lines which are connected to the nucleon source, as well as those where the operator acts on a quark loop in the vacuum, which is connected only by gluon lines to the quark lines originating in the nucleon source. Obtaining a signal from the latter, so-called "disconnected graphs" is extremely difficult on the lattice, as recent studies 18 of the strangeness form factors of the nucleon have shown, and to date only the connected contributions to the lattice PDF moments have been computed. Fortunately, the near degeneracy of the $u$ and $d$ quark masses, which makes charge symmetry such a good symmetry of nature, means that the disconnected diagrams cancel very precisely in the isovector difference between the $u$ and $d$ PDFs of the proton. An unambiguous comparison of the connected isovector PDF lattice moments can therefore be made with the analogous experimental moments.

The mass dependence of the moments of the unpolarized isovector PDF of the nucleon can be parameterized as 16

$$
\left\langle x^{n}\right\rangle_{u-d}=a_{n}\left(1+c_{\mathrm{LNA}} m_{\pi}^{2} \log \frac{m_{\pi}^{2}}{m_{\pi}^{2}+\mu^{2}}\right)+b_{n} \frac{m_{\pi}^{2}}{m_{\pi}^{2}+m_{b, n}^{2}},
$$

where (for $n>0$ ) the chiral coefficient is given by $\frac{1415}{15 N A} c_{\text {LNA }}=-\left(1+3 g_{A}^{2}\right) /\left(4 \pi f_{\pi}\right)^{2}$. Although we shall work only with full QCD, we note that the corresponding coefficients have also been computed in quenched and partially-quenched chiral perturbation theory 19 The $n=0$ moment, which corresponds to isospin charge, is not renormalized by pion loops. The argument of the logarithm corresponds to the case where the pion loops are regulated with a simple, sharp three-momentum cut-off. The parameter $\mu$ has the effect of suppressing the rapid variation of the chiral logarithm away from the chiral limit. Physically it is related to the size of the nucleon core, which acts as the source of the pion field 12 Since the isovector distribution $u(x)-d(x) \rightarrow \delta(x-1 / 3)$ in the heavy quark limit (i.e., as $\left.m_{q} \rightarrow \infty\right)$, one may choose to constrain the constant $b_{n}$ in Eq. (4) by

$$
b_{n}=\frac{1}{3^{n}}-a_{n}\left(1-\mu^{2} c_{\mathrm{LNA}}\right) .
$$

The results of the fits to the lattice data for the $n=1,2$ and 3 moments of the unpolarized $u-d$ distribution using Eq. (4) are shown in Fig. 1. In each plot the central curve is shown with two error bands: the inner (darkly shaded) band shows variation of $\mu$ by $\pm 20 \%$, whilst the outer (lightly shaded) shows the additional effects that result from shifting the lattice data up and down within the extent of their error bars. The extrapolated moments at the physical pion mass are listed in Table 1 The fits to the data are quite insensitive to the choice of $m_{b, n}$ (as long as it is large), $\frac{16}{1}$ and it has been set to $5 \mathrm{GeV}$ for all $n$. In practice, fits to the lattice data in which the heavy quark limit is not guaranteed (i.e., where the term involving 


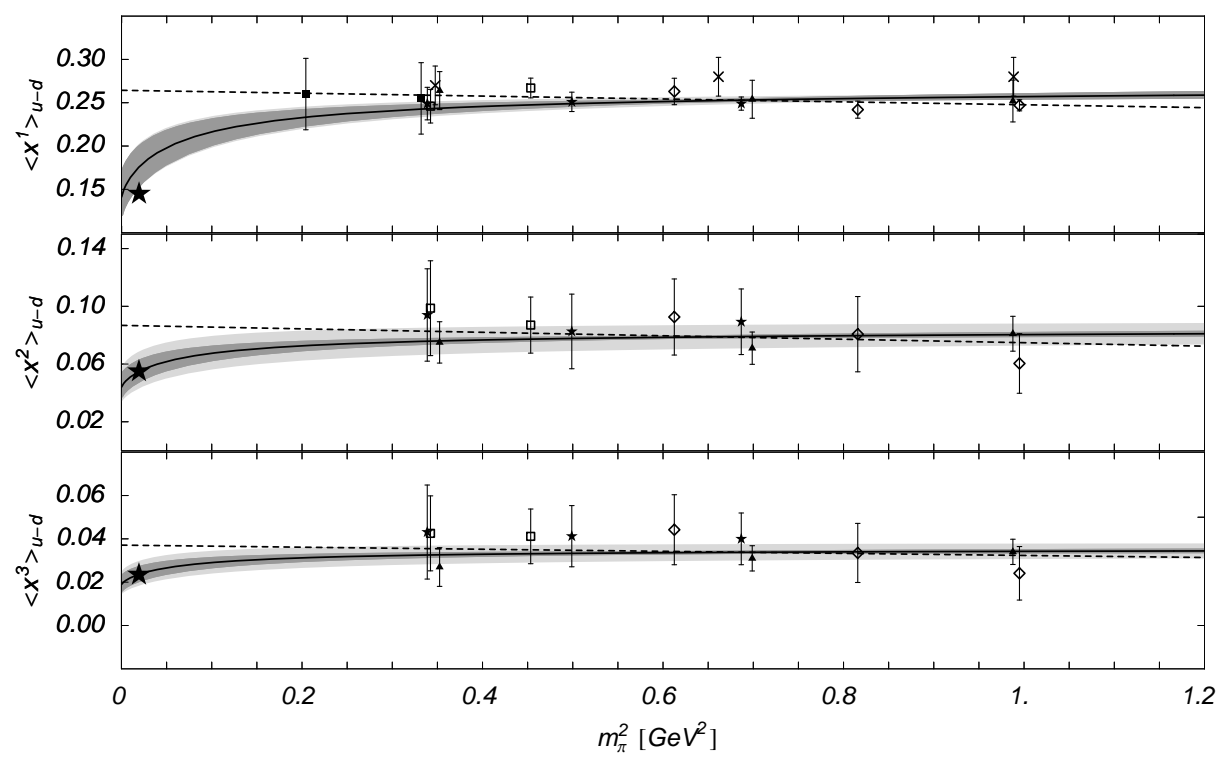

Fig. 1. Moments of the unpolarized $u-d$ distribution in the proton, for $n=1,2$ and 3. Lattice data 10 include both quenched (solid symbols) and unquenched (open symbols) results. The solid line represents the full chiral extrapolation, while the inner (darkly shaded) error band shows variation of $\mu$ by $\pm 20 \%$, with the outer band (lightly shaded) showing the additional effects of shifting the lattice data within the extent of their error bars. Linear extrapolations are indicated by dashed lines, and the phenomenological values 20 are shown as large stars at the physical pion mass.

$b_{n}$ is simply $\left.b_{n} m_{\pi}^{2}\right)$ and $b_{n}$ is a third fitting parameter, 7 are indistinguishable from those in Fig. 1.

Note that the majority of the data points (filled symbols) are obtained from simulations employing the quenched approximation (in which background quark loops are neglected) whereas Eq. (4) is based on full QCD with quark loop effects included. On the other hand, recent calculations with dynamical quarks suggest that at the relatively large pion masses $\left(m_{\pi}>0.5-0.6 \mathrm{GeV}\right)$ where the full simulations are currently performed, the effects of quark loops are largely suppressed, as the data in Fig. 1 (small open symbols) indicate. Further details of the lattice data, 231415 and a more extensive discussion of the fit parameters, can be found elsewhere.10

A similar analysis leads to analogous lowest order LNA parameterizations of the mass dependence of the spin-dependent moments 17

$$
\left\langle x^{n}\right\rangle_{\Delta u-\Delta d}=\Delta a_{n}\left(1+\Delta c_{\mathrm{LNA}} m_{\pi}^{2} \log \frac{m_{\pi}^{2}}{m_{\pi}^{2}+\mu^{2}}\right)+\Delta b_{n} \frac{m_{\pi}^{2}}{m_{\pi}^{2}+m_{b, n}^{2}},
$$

and

$$
\left\langle x^{n}\right\rangle_{\delta u-\delta d}=\delta a_{n}\left(1+\delta c_{\mathrm{LNA}} m_{\pi}^{2} \log \frac{m_{\pi}^{2}}{m_{\pi}^{2}+\mu^{2}}\right)+\delta b_{n} \frac{m_{\pi}^{2}}{m_{\pi}^{2}+m_{b, n}^{2}},
$$


Table 1. Experimental moments 2021 and moments extrapolated 10 from lattice data.

\begin{tabular}{|c|cc|}
\hline \hline Moment & Experiment & Extrapolated \\
\hline \hline$\left\langle x^{1}\right\rangle_{u-d}$ & $0.145(4)$ & $0.18(3)$ \\
$\left\langle x^{2}\right\rangle_{u-d}$ & $0.054(1)$ & $0.05(2)$ \\
$\left\langle x^{3}\right\rangle_{u-d}$ & $0.022(1)$ & $0.02(1)$ \\
$\left\langle x^{0}\right\rangle_{\Delta u-\Delta d}$ & $1.267(4)$ & $1.12(6)$ \\
$\left\langle x^{1}\right\rangle_{\Delta u-\Delta d}$ & $0.210(25)$ & $0.27(2)$ \\
$\left\langle x^{2}\right\rangle_{\Delta u-\Delta d}$ & $0.070(11)$ & $0.14(4)$ \\
$\langle 1\rangle_{\delta u-\delta d}$ & - & $1.23(7)$ \\
$\langle x\rangle_{\delta u-\delta d}$ & - & $0.51(9)$ \\
\hline \hline
\end{tabular}

where the LNA coefficients are given by $\Delta c_{\mathrm{LNA}}=-\left(1+2 g_{A}^{2}\right) /\left(4 \pi f_{\pi}\right)^{2}$ and $\delta c_{\mathrm{LNA}}=-\left(1+4 g_{A}^{2}\right) / 2\left(4 \pi f_{\pi}\right)^{2}$. In the heavy quark limit, where spin-flavor symmetry is exact, both $\Delta u(x)-\Delta d(x)$ and $\delta u(x)-\delta d(x)$ are given by $22 \frac{5}{3} \delta(x-1 / 3)$, which leads to the constraints

$$
\Delta b_{n}=\frac{5}{3^{n+1}}-\Delta a_{n}\left(1-\mu^{2} \Delta c_{\mathrm{LNA}}\right)
$$

and

$$
\delta b_{n}=\frac{5}{3^{n+1}}-\delta a_{n}\left(1-\mu^{2} \delta c_{\mathrm{LNA}}\right) .
$$

These are the most general lowest order parameterizations of the twist-2 PDF moments consistent with chiral symmetry and the heavy quark limits of QCD.

The $n=0$ moment of the spin-dependent isovector PDF is equivalent to the axial charge of the nucleon, $g_{A}$. It is well known, 23 for instance through the AdlerWeisberger sum rule, that the $\Delta$ resonance plays a key role in $g_{A}$. In the framework of the chiral expansion, vertex renormalization contributions involving a $\Delta$ isobar, although not leading non-analytic, are large and extremely important in countering the effect of wave function renormalization. 1024 Indeed, it is entirely because of the explicit role of the $\Delta$ that the bare and renormalized $\pi N N$ couplings are close. $\frac{25}{}$

From the point of view of the chiral extrapolation problem, the tendency for cancellation between wave function and polarized vertex corrections means that, once virtual $\pi \Delta$ loops are included, the chiral variation as $m_{\pi} \rightarrow 0$ is approximately linear. That is, for the polarized moments the chiral non-analytic behavior produces far less curvature as one approaches the physical pion mass than in the unpolarized case. This can be seen explicitly in Figs. 2 and 3, where the $n=0,1$ and 2 moments of the helicity distribution $\Delta u(x)-\Delta d(x)$ and the $n=0$ and 1 moments of the transversity distribution $\delta u(x)-\delta d(x)$ are plotted, respectively. In these figures, the long-dashed curves show the extrapolations ignoring the $\pi \Delta$ contributions.

The curvature in the extrapolated lowest moments of the helicity distribution $\left(g_{A}\right)$ increases somewhat the discrepancy between the lattice value and experiment, although the contributions of the $\Delta$ largely reduce this effect. Nevertheless, there does appear to be a residual $10-15 \%$ underestimation of $g_{A}$, while at the same 


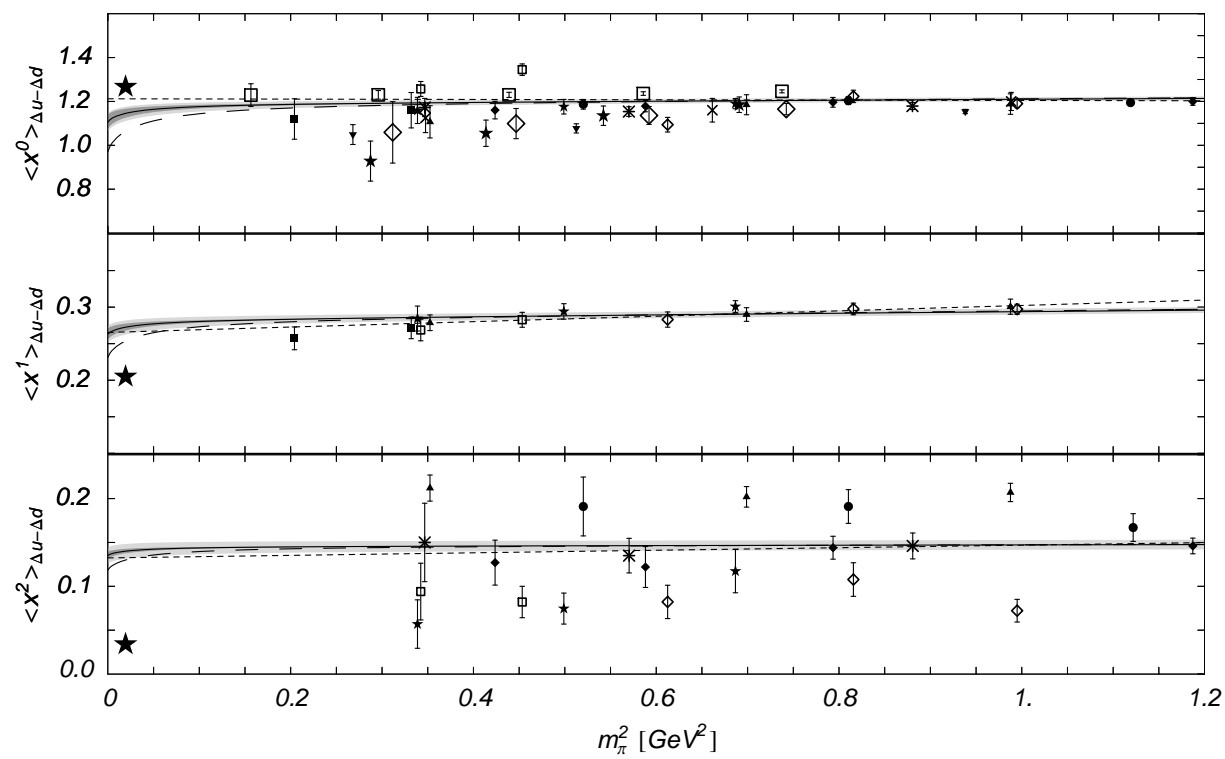

Fig. 2. Moments of the helicity distribution $\Delta u-\Delta d$ in the nucleon for $n=0,1$ and 2. The long-dashed curves show extrapolations neglecting the $\Delta$. The meaning of all other symbols is as in Fig. 1.

time the $n=1$ and 2 moments of $\Delta u-\Delta d$ are somewhat overestimated (although the errors on the $n=2$ lattice data in particular are rather large). There have been several suggestions for the possible origin of this discrepancy, including the effects of working with a finite volume on the lattice. The RBCK Collaboration has in fact observed an unusually strong dependence of $g_{A}$ on the lattice volume. This is illustrated in the top panel of Fig. 2 where the large open boxes and diamonds correspond to calculations on lattices with spatial volumes of $(2.4 \mathrm{fm})^{3}$ and $(1.2 \mathrm{fm})^{3}$, respectively. Clearly the issue of understanding the value of $g_{A}$ in QCD is of central importance in hadronic physics; moreover, resolution of this apparent discrepancy should enable a more reliable prediction to be made for the moments of the transversity distribution in Fig. 3, for which there are as yet no experimental data.

\subsection{Volume dependence}

The origin of the strong lattice volume dependence of $g_{A}$ is likely related to the fact that the pion field does not vanish before it meets the lattice boundary! 26 However, there is another important issue to consider when it comes to the curvature that we have been discussing with regard to the chiral extrapolation problem. One would really like to see confirmation of this curvature as the lattice calculations are continued to smaller pion mass and eventually use these calculations to determine 


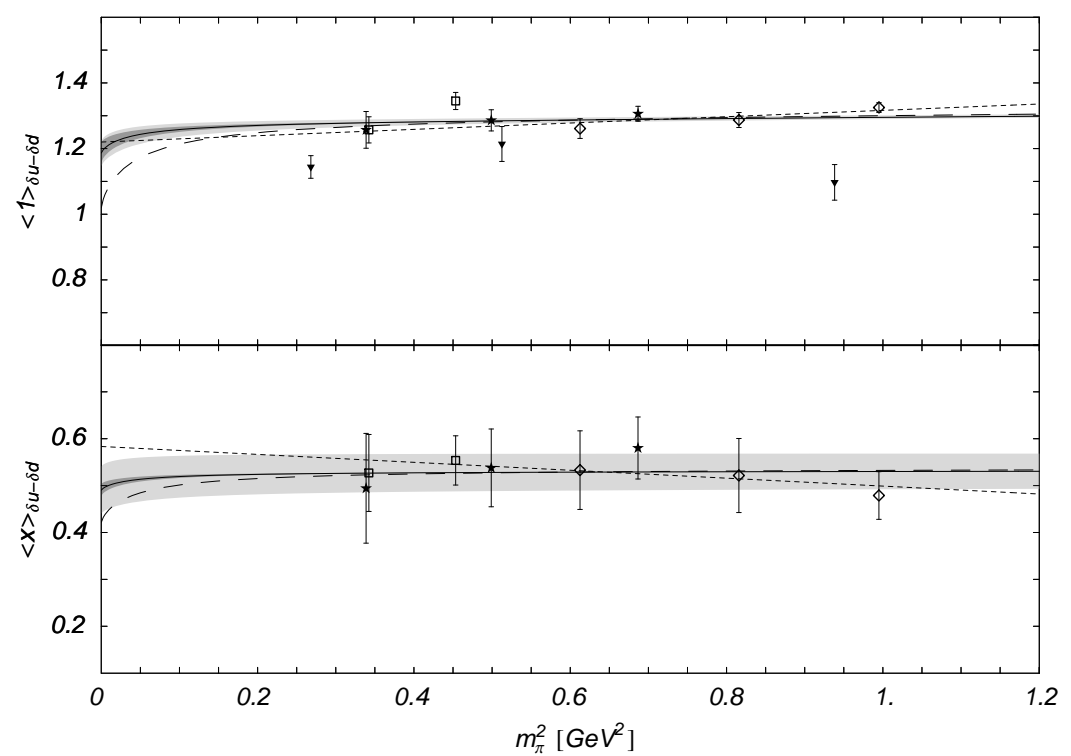

Fig. 3. Moments of the isovector transversity distribution $\delta u-\delta d$ for $n=0$ and 1. Symbols and curves are as in Fig. 2.

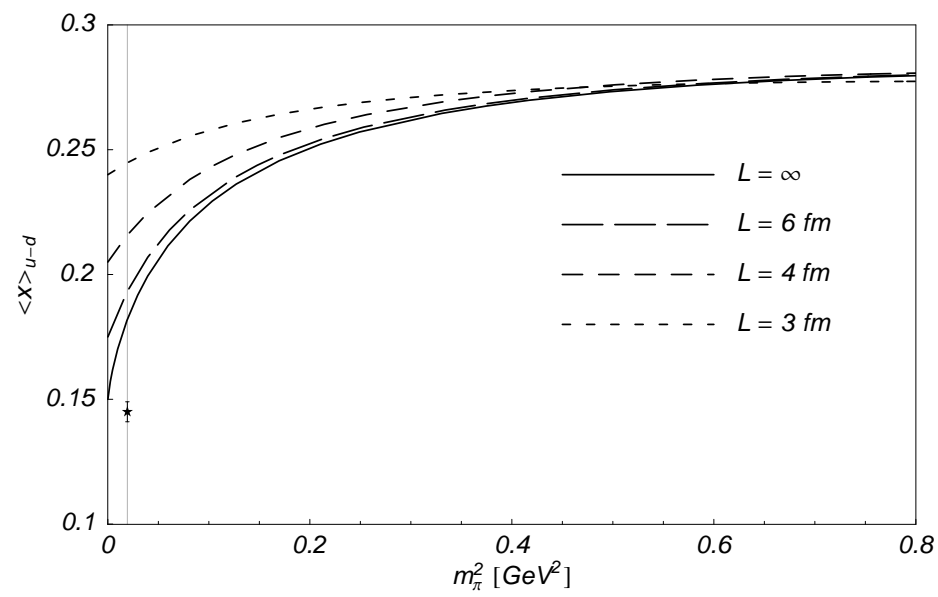

Fig. 4. Estimate of the volume dependence of the chiral curvature in the extrapolation of the isovector quark momentum fraction, $\langle x\rangle_{u-d}$, obtained with a simple cut-off on the lower limit of the pion three momentum, for various box side lengths $L$.

the parameter $\mu$. This is difficult for two reasons. First, the chiral coefficients are smaller in quenched simulations (which are numerically less demanding), and so the curvature may not be observable until very low masses are reached. Secondly, even in the case of full QCD the chiral behavior, which is an infrared property of QCD, 
can be severely altered by working on a finite volume.

For an accurate estimate of the effect of a finite lattice volume on the chiral loops one should replace the continuum momentum integrals in the chiral expansion by finite sums over the allowed momenta on the lattice - as already done for baryon and vector meson masses. 27 A full presentation of the results of such an approach will be given elsewhere ${ }^{28}$ For the present we note that the main consequence of a finite volume is to effectively impose a lower limit on the pion momentum integral. One can estimate the effect very simply by replacing the factor $m_{\pi}^{2} /\left(m_{\pi}^{2}+\mu^{2}\right)$ in Eq. (4) by $\left(m_{\pi}^{2}+\mathcal{L}^{2}\right) /\left(m_{\pi}^{2}+\mu^{2}\right)$, where $\mathcal{L}=2 \pi / N a \equiv 2 \pi / L$ is the lowest non-zero momentum available to a pion on the lattice, with $a$ the lattice spacing and $N$ the number of lattice sites in each spatial dimension (i.e., a lattice of side $L \mathrm{fm}$ ). The results of such a simple calculation are illustrated in Fig. 4 from which we conservatively estimate that one may need a box $L \sim 4 \mathrm{fm}$ on a side to see substantial chiral curvature in the extrapolation, even in full QCD.

\section{Bjorken-x dependence of quark distributions}

In this section we present the results of recent efforts to reconstruct the $x$ dependence of the quark distributions from their extrapolated moments. We shall briefly present updated results for the unpolarized isovector distributions in the nucleon, and then focus on new results for the longitudinally polarized valence quark distribution. An analogous investigation of the valence quark distribution in the pion was

performed recently, ${ }^{8}$ whilst analysis of transversely polarized distributions awaits the calculation of additional moments.

\subsection{Reconstruction method}

Although the Mellin transforms, Eqs. (1)-(3), have mathematically well-defined inverses, such inversion requires knowledge of the behavior of the moments along a contour in the complex- $n$ plane. Clearly, evaluation of nucleon matrix elements of twist-2 operators (on the lattice or elsewhere) cannot provide this - through the OPE, appropriate matrix elements determine moments only for real, integral $n$. In order to proceed, one must assume a parametric structure of the underlying parton distribution. This, however, is not a new obstacle; the same problem is encountered in parameterizations of PDFs from experimental data at different scales and quark distribution functions $f(x)$ (polarized and unpolarized) are often parameterized with the form

$$
x f(x)=A x^{b}(1-x)^{c}(1+\epsilon \sqrt{x}+\gamma x) .
$$

The parameters $b$ and $c$ determine the small- and large- $x$ behavior, respectively, and have physical interpretations in terms of Regge behavior and counting rules, whereas $\epsilon$ and $\gamma$ are introduced to provide additional freedom. One easily sees that 
the corresponding Mellin moments are given in terms of the $\beta$-function by

$$
\left\langle x^{n}\right\rangle_{f}=A\left[B(1+c, b+n)+\epsilon B\left(1+c, \frac{1}{2}+b+n\right)+\gamma B(1+c, 1+b+n)\right] .
$$

Given this parametric form, one can now use the various sets of moments extrapolated from the lattice data to fit the parameters in Eq. (11). Since there are at most only three nontrivial moments available for each distribution, one must first reduce the number of free parameters by fitting $\epsilon$ and $\gamma$ to an average of the available unpolarized 20 and polarized 21 phenomenological parameterizations. This leaves only the parameters $A, b$ and $c$ to be determined from the lattice data. If one omits $\epsilon$ and $\gamma$, it is not clear that $b$ and $c$ retain their physical meanings since there is no longer enough freedom in the fits for these parameters to be determined solely by the small- and large- $x$ regions, respectively. This is evident when one calculates the moments of a known distribution and then attempts to use Eq. (11) to reconstruct the distribution $f(x)$. By studying the dependence of the reconstructed parameters on the number of moments, $N$, used in the fit it is apparent that in the full fit, Eq. (11), the parameters $A, b$ and $c$ are almost independent of $N$, while $\epsilon$ and $\gamma$ show some variation. On the other hand, if $\epsilon=\gamma=0$, the remaining (purportedly physical) parameters show significantly more variation.

As mentioned in Sec. 2 above, the different crossing symmetry properties of the even and odd moments ( $c f$. Eqs. (1)-(3) mean that the lattice data correspond to valence (odd under charge conjugation, $C$ ) moments for $n$-even ( $n$-odd) in the case of the unpolarized and transversity (helicity) distributions, and to the total (even under $C$ ) moments for the remaining cases. Ideally, one would like several moments for each of the $C$-odd and $C$-even distributions to reconstruct both the valence and the total distributions (which would then allow one to determine the sea). In practice, not enough moments are currently known from the lattice, and one must be content with reconstructing the valence distributions with the help of additional phenomenology. Namely, to recover the valence moments, one needs to shift the relevant extrapolated lattice moments by the corresponding moments of the sea quark distribution. In practice, since sea quarks are concentrated at small $x$, it is only the lowest moments ( $n=0$ in the polarized case, $n=1$ for unpolarized) that shift to any appreciable degree. For the unpolarized case, the non-singlet sea $\bar{d}-\bar{u}$ is well determined from Drell-Yar $\frac{29}{29}$ and deep inelastic data, ${ }^{[30}\langle x\rangle_{\bar{d}-\bar{u}}=0.008(1)$. On the other hand, the corresponding quantities for the polarized distributions are poorly known and estimates need to be taken into account in the analysis.

\subsection{Reconstructed distributions}

The unpolarized valence isovector distribution reconstructed 16 from the lattice moments is shown in Fig. 5 (solid line). To present realistic error bands on the reconstructed distribution, a large ensemble (of order 200 elements) of sets of moments is generated by randomly varying each moment within the extrapolated lattice error bars. The lightly shaded region then corresponds to the envelope of these 


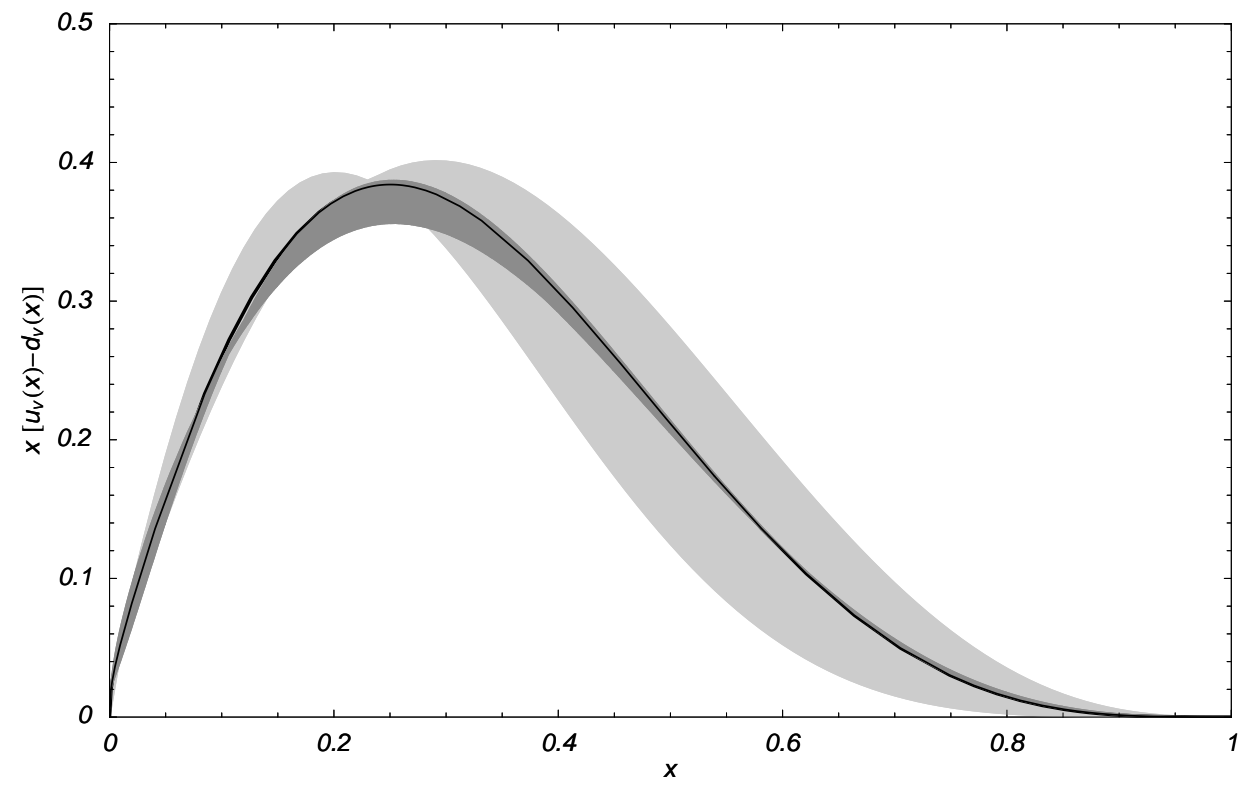

Fig. 5. Reconstructed isovector valence quark distribution $x\left(u_{v}-d_{v}\right)$ in the proton at $Q^{2}=$ $4 \mathrm{GeV}^{2}$. The central fit curve (solid line) and error band (lightly shaded) are compared with the envelope of the phenomenological distributions 20 (darkly shaded).

distributions (the humps in the error band arise from taking the extrema of the distributions). The parameter values and errors are quoted in Table 2 and are given by the mean and standard deviation over the ensemble. The darkly shaded region corresponds to the average of (next-to-leading order) PDF parameterizations 20 of $u_{v}(x)-d_{v}(x)$ at $Q^{2}=4 \mathrm{GeV}^{2}$. The agreement between the reconstructed distributions and the phenomenological parameterizations is clearly excellent.

Table 2. Fit parameters for the reconstructed distributions. For each polarized data set, the upper (lower) rows show the parameters for fits with $b(c)$ fixed to its phenomenological value. In the unpolarized (polarized) fits, $\epsilon=1.96$ and $\gamma=29.1(\epsilon=-0.63$ and $\gamma=10.1)$ were set to their phenomenological values. 2021

\begin{tabular}{|c|ccc|}
\hline \hline Data set & $A$ & $b$ & $c$ \\
\hline \hline Unpolarized & $0.21(5)$ & $-0.63(3)$ & $3.8(6)$ \\
\hline Polarized, set I & $0.47(3)$ & -0.51 & $2.1(2)$ \\
& $1.9(2)$ & $-0.05(7)$ & 3.69 \\
\hline Polarized, set II & $0.28(5)$ & -0.51 & $1.4(2)$ \\
& $5(2)$ & $0.8(5)$ & 3.69 \\
\hline Polarized, set III & $0.61(4)$ & -0.51 & $2.5(1)$ \\
& $1.5(1)$ & $-0.24(6)$ & 3.69 \\
\hline Polarized, set IV & $0.70(5)$ & -0.51 & $3.0(2)$ \\
& $1.1(1)$ & $-0.39(5)$ & 3.69 \\
\hline \hline
\end{tabular}


In contrast to the unpolarized distribution, there are a number of issues that complicate the analysis of the polarized distributions. Firstly, from the crossing symmetry properties of spin-dependent structure functions, the $n=0$ moments extracted from the lattice correspond to moments of the total distribution, $\Delta q(x)+\Delta \bar{q}(x)$, and in order to construct the valence moment one must subtract (twice) the polarized sea moment. Unfortunately, the only experimental information on $\langle 1\rangle_{\Delta \bar{u}-\Delta \bar{d}}$ from the HERMES data 31 does not provide strong constraints. Consequently, we shall investigate two scenarios: $\langle 1\rangle_{\Delta \bar{u}-\Delta \bar{d}}=0$ (as suggested in models in which the non-perturbative sea is generated through meson loops 32 ), and $\langle 1\rangle_{\Delta \bar{u}-\Delta \bar{d}}=0.2(2)$ (as in quark models with SU(6) symmetry, when the Pauli exclusion principle is applied $\frac{33}{3}$.
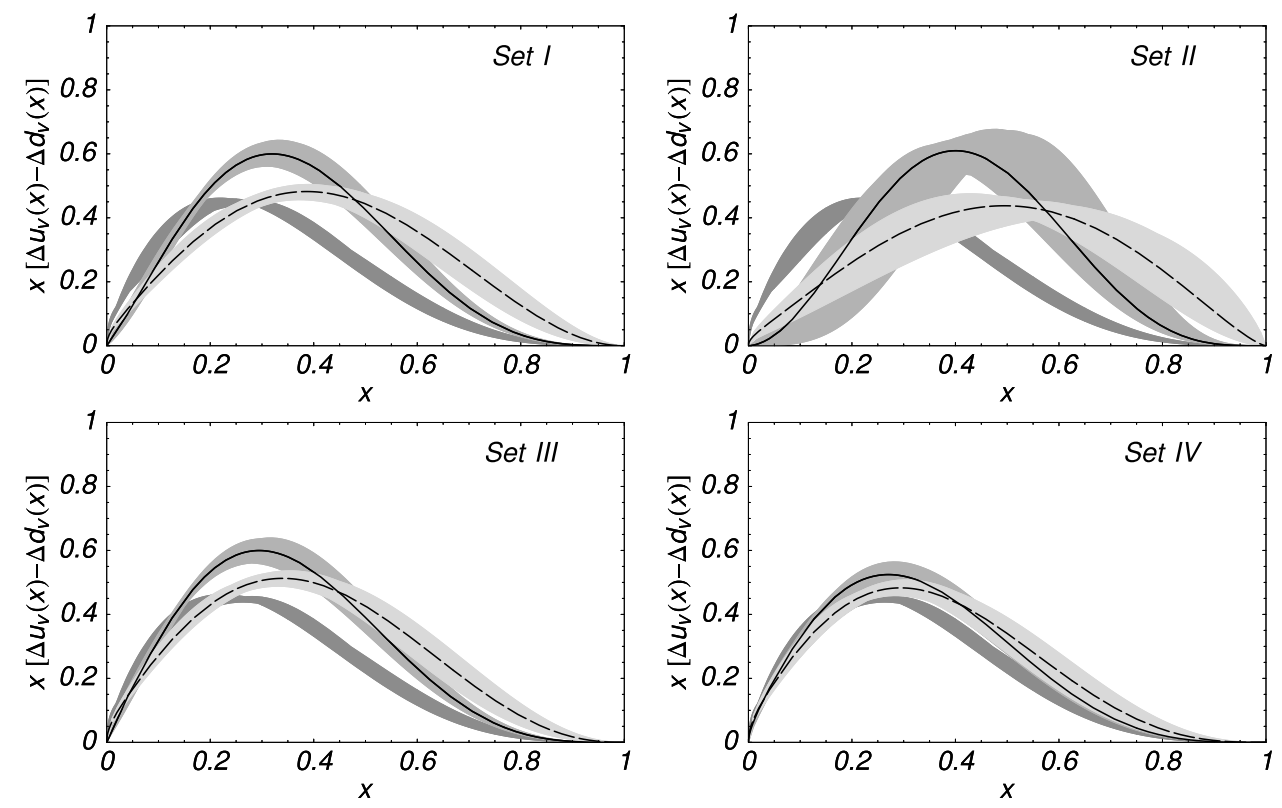

Fig. 6. Reconstructed isovector valence helicity distribution, $x\left(\Delta u_{v}-\Delta d_{v}\right)$ at $Q^{2}=4 \mathrm{GeV}^{2}$. In each panel, the solid (dashed) curve and the corresponding error band is a best fit with $b(c)$ in Eq. (10) fixed, while the darkly shaded region corresponds to the envelope of the phenomenological distributions. 21 The four sets of curves are described in the text.

Secondly, as discussed above, finite volume effects have been found $\frac{5}{5}$ to be particularly significant in lattice calculations of $g_{A}=\langle 1\rangle_{\Delta u-\Delta d}$, and to account for these we shall consider the effects of shifting the extrapolated moments towards their physical value. Finally, fitting the three parameters $A, b$ and $c$ with only three moments is unreliable (though possible), so one must presently set either $b$ or $c$ to its phenomenological value.21

In Fig. 6 we show the reconstructed PDFs for various shifting scenarios compared with phenomenological parameterizations. Each panel shows the fits and error 
bands with $b$ fixed (solid curve, medium shading), and with $c$ fixed (long-dashed curve, light shading). The darkly shaded region corresponds to the envelope of the phenomenological distributions. ${ }^{21}$ In the upper left panel, the unmodified extrapolated lattice moments are used (data set I). Fits to these with either $b$ or $c$ fixed give a somewhat different reconstructed distribution, and the PDFs extracted from the lattice are not in agreement with the phenomenological fits.

In the upper right plot we show the resulting fits that arise from shifting the $n=0$ moment up by $0.2(2)$ to correct for the sea contribution which enters the lattice extrapolated moment (data set II). The agreement between the two fits (with $b$ and $c$ held fixed) is still poor and the errors are considerably increased. In the lower panels, we contemplate shifts of the moments to correct for possible finite volume (and other) effects in the lattice data and their extrapolations. On the left, we shift just the $n=0$ moment, $g_{A}$, so that it agrees with experiment (data set III), while on the right all three moments are shifted by the same relative percentage towards the experimental moments (data set IV). The latter is the best case scenario, with the agreement between the two reconstructed distributions significantly improved, and (not surprisingly) in excellent agreement with the phenomenological fits. The parameters for the various fits are given in Table2] From the variations in Fig. [6] one can conclude that the lattice data currently limit the accuracy of the reconstructed $x$ dependence of the isovector valence helicity distribution, and that it is vital to understand the current discrepancy between the lattice and experimental values of $g_{A}$. We shall discuss the necessary improvements in Sec. 4 .

\subsection{Quark mass dependence of $x$ distributions}

In the moment analyses in Sec. 2 the most dramatic variation with $m_{\pi}$ of the moments occurs at small $m_{\pi}$ values, as one approaches the chiral limit. The transition to the heavy quark limit, on the other hand, appears to have little visible effect on the moments, and in practice one obtains essentially identical fits to the lattice data whether one explicitly imposes the heavy quark limit or not.

A more graphic illustration of the change of the structure of the nucleon from the physical region to the heavy quark limit is provided by the quark mass dependence of the $x$ distributions. The chiral extrapolation formulas of Sec. 2 provide moments for any value of $m_{\pi}$, which allows one to trace how the $x$ dependence changes as one goes from the chiral limit to the heavy quark limit, where the distribution approaches a $\delta$-function.

As an example, we consider the unpolarized valence $u_{v}-d_{v}$ distribution, which we plot in Fig. 17 for various pion masses from $m_{\pi}=0$ to $m_{\pi}=5 \mathrm{GeV}$. As $m_{\pi}$ increases, the distribution becomes more sharply peaked, with the peak moving towards the limiting value of $x=1 / 3$. This corresponds to fit parameters $b$ and $c$ becoming larger, as the $x$ dependence of the valence distribution becomes less singular at small $x$. The distribution at $m_{\pi}=5 \mathrm{GeV}$ is already rather steep, and resembles a constituent quark-like distribution, peaking near $x \sim 1 / 3$. Interestingly, 


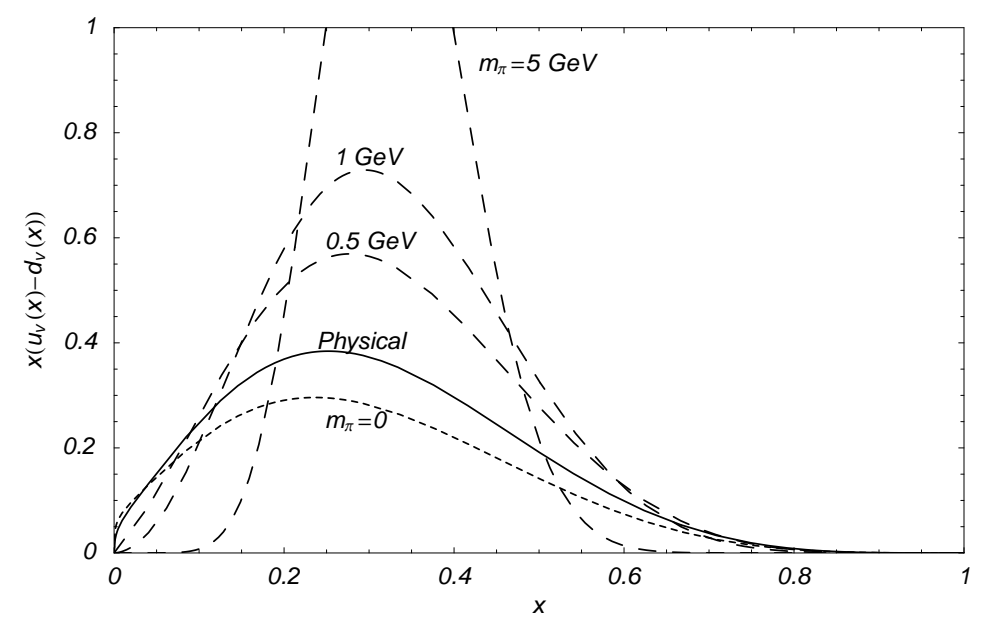

Fig. 7. Dependence of the isovector unpolarized valence quark distribution on the pion mass.

the shape of this distribution is very similar to that extracted from the moments extrapolated linearly to the physical pion mass 16 A similar analysis can in principle also be performed for polarized PDFs, although the present uncertainties in the $m_{\pi}$ dependence of the polarized lattice moments and the polarized sea prohibit a reliable reconstruction of the $x$ dependence as a function of $m_{\pi}$.

\section{Conclusions}

The study of the PDFs of the nucleon using lattice QCD has already achieved several notable successes. In the unpolarized case the level of agreement between the first three non-trivial moments of the isovector distribution and experiment is excellent. To realize this level of agreement one must incorporate the correct leading non-analytic behavior of chiral perturbation theory into the extrapolation of the moments from the relatively large masses accessible in current lattice QCD simulations to the physical regime. Given the normalization condition and an additional three moments one can reconstruct the $x$ dependence of the isovector valence PDF quite reliably and, as shown in Fig. 5] the agreement with experiment is quite impressive.

The analysis presented here for the polarized isovector quark distribution highlights a number of successes and also areas which demand further study. Amongst the successes we note:

- There has been a clear demonstration of the rather unusual degree of sensitivity of the polarized moments to the volume of the lattice. Moreover, in the case of $g_{A}$, the agreement with the experimental value definitely improves as the volume increases. 5

- Studies of the chiral extrapolation procedure have clearly established the need to include virtual $\Delta$ isobars! 1024 Once the $\Delta$ is included there is a 
significant reduction in the chiral curvature as $m_{\pi} \rightarrow 0$, compared with that found in the unpolarized case.

For the unpolarized and especially the polarized PDFs there are a number of challenges which must be answered in order to reach the stage at which lattice calculations rival experimental determinations in their accuracy. In particular:

- In order to better constrain the chiral extrapolations it is important to push the lattice simulations to lower pion masses. Ideally this should occur in full (unquenched) QCD, however, quenched, and especially partially-quenched simulations would also provide valuable information to guide the extrapolation. For the unpolarized isovector case, $u-d$, one needs to confirm the predicted chiral curvature of the moments at low pion mass. As suggested by the estimates shown in Fig. 4 this will most likely require high statistics simulations on lattices of dimensions $L \sim 4 \mathrm{fm}$ at a pion mass of order $300 \mathrm{MeV}$ or lower.

- To better constrain the functional form of the $x$-dependence of the PDFs, calculations of several higher moments (e.g. $n=3,4,5)$ are necessary. This is particularly relevant in the polarized case. Such calculations would necessitate the non-perturbative calculation of operator mixing coefficients.

- Finite volume effects must be explored more thoroughly and taken into account in future extrapolations. 28 The effects of a finite lattice spacing $(a \neq 0) \sqrt{34}$ must also be incorporated.

- In the isoscalar case, one has to resolve the numerical problem of obtaining a reliable signal for the contribution from disconnected quark loops. Furthermore, we note that separations of the sea quark distributions from the total isoscalar PDFs, based on lattice data alone, have not yet been attempted. They will also require an accurate knowledge of moments with $n>3$ and evaluation of disconnected contributions.

With such a program we could expect significant advances in our understanding of the quark structure of the nucleon (and other hadrons) over the next few years. In particular, we look forward to determinations of the various sea and transversity distributions. The methods reviewed here will also prove useful in extracting gener-

alized parton distributions from recent lattice calculations 35 of non-forward matrix elements of the various twist-2 operators.

\section{Acknowledgments}

This work was supported by the Australian Research Council, the U.S. Department of Energy contract DE-FG03-97ER41014 and contract DE-AC05-84ER40150, under which the Southeastern Universities Research Association (SURA) operates the Thomas Jefferson National Accelerator Facility (Jefferson Lab). 


\section{References}

1. G. Martinelli and C. T. Sachrajda, Phys. Lett. B 196, 184 (1987); ibid B 217, 319 (1989).

2. D. Dolgov et al., Phys. Rev. D 66, 034506 (2002).

3. M. Göckeler et al., Phys. Rev. D 53, 2317 (1996); Nucl. Phys. Proc. Suppl. 53, 81 (1997); Nucl. Phys. A711, 291 (2002); S. Capitani et al., Nucl. Phys. Proc. Suppl. 106, 299 (2002); C. Best et al., hep-ph/9706502

4. M. Fukugita et al., Phys. Rev. Lett. 75, 2092 (1995); S. Aoki et al., Phys. Rev. D 56, 433 (1997).

5. S. Sasaki, K. Orginos, S. Ohta and T. Blum, hep-lat/0306007 Nucl. Phys. Proc. Suppl. 106, 302 (2002).

6. A.W. Thomas, Nucl. Phys. Proc. Suppl. 119, 50 (2003).

7. W. Detmold et al., Phys. Rev. Lett. 87, 172001 (2001).

8. W. Detmold, W. Melnitchouk and A. W. Thomas, Phys. Rev. D 68, 034025 (2003).

9. M. Göckeler et al., Phys. Rev. D 54, 5705 (1996).

10. W. Detmold, W. Melnitchouk and A.W. Thomas, Phys. Rev. D 66, 054501 (2002).

11. A.W. Thomas, Adv. Nucl. Phys. 13, 1 (1984).

12. W. Detmold et al., Pramana 57, 251 (2001).

13. A.W. Thomas, W. Melnitchouk and F.M. Steffens, Phys. Rev. Lett. 85, 2892 (2000).

14. D. Arndt and M.J. Savage, Nucl. Phys. A697, 429 (2002).

15. J.W. Chen and X. Ji, Phys. Lett. B 523, 107 (2001).

16. W. Detmold, W. Melnitchouk, and A.W. Thomas, Eur. Phys. J. direct C 3, 13 (2001).

17. W. Detmold, W. Melnitchouk and A.W. Thomas, Int. J. Mod. Phys. A 18, 1343 (2002); hep-lat/0210029

18. R. Lewis, W. Wilcox and R. M. Woloshyn, Phys. Rev. D 67, 013003 (2003).

19. J.W. Chen and M.J. Savage, Nucl. Phys. A707, 452 (2002); Phys. Rev. D 65, 094001 (2002); S.R. Beane and M.J. Savage, Nucl. Phys. A709, 319 (2002).

20. H.L. Lai et al., Eur. Phys. J. C 12, 375 (2000); A.D. Martin et al., Eur. Phys. J. C 14, 133 (2000); M. Glück, E. Reya and A. Vogt, Eur. Phys. J. C 5, 461 (1998).

21. Y. Goto et al., Phys. Rev. D 62, 034017 (2000); J. Blümlein and H. Böttcher, Nucl. Phys. B636, 225 (2002); D. de Florian and R. Sassot, Phys. Rev. D 62, 094025 (2000); E. Leader et al., Eur. Phys. J. C 23, 479 (2002); M. Glück et al., Phys. Rev. D 63, 094005 (2001); T. Gehrmann and W.J. Stirling, Phys. Rev. D 53, 6100 (1996).

22. M. Göckeler et al., Nucl. Phys. Proc. Suppl. 49, 250 (1996).

23. See e.g. A.W. Thomas and W. Weise, The Structure Of The Nucleon (Wiley-VCH, Berlin, Germany, 2001).

24. T.R. Hemmert, M. Procura and W. Weise, hep-lat/0303002

25. S. Theberge, G.A. Miller and A.W. Thomas, Can. J. Phys. 60, 59 (1982); L.R. Dodd, A.W. Thomas and R.F. Alvarez-Estrada, Phys. Rev. D 24, 1961 (1981).

26. R. L. Jaffe, Phys. Lett. B 529, 105 (2002).

27. R.D. Young et al., Phys. Rev. D 66, 094507 (2002); D.B. Leinweber et al., Phys. Rev. D 64, 094502 (2001).

28. W. Detmold et al., in preparation.

29. R.S. Towell et al., Phys. Rev. D 64, 052002 (2001).

30. M. Arneodo et al., Phys. Rev. D 50, 1 (1994); K. Ackerstaff et al., Phys. Rev. Lett. 81, 5519 (1998).

31. A. Airapetian et al., hep-ex/0307064

32. A.W. Thomas, Phys. Lett. B 126, 97 (1983).

33. A.W. Schreiber, A.I. Signal and A.W. Thomas, Phys. Rev. D 44, 2653 (1991). 
Extraction of parton distributions from lattice QCD 17

34. S. R. Beane and M. J. Savage, hep-lat/0306036

35. P. Hägler et al., Phys. Rev. D 68, 034505 (2003); M. Göckeler et al., hep-ph/0304249 